\title{
Who'd want to work in a team?
}

Biologists and their institutions are increasingly confronted by the challenges of working in major collaborations that other disciplines have already addressed. A gathering last week showed how much further there is to go.

$\mathrm{T}$ eam science is everywhere these days. The trouble is, you'd never guess it from an inspection of the universities that house it or the agencies that fund and supposedly foster it. Last week, a meeting at the US National Institutes of Health (NIH) on "Catalyzing team science" highlighted the difficulties, and proposed some solutions. What should disturb everybody is how far from reality many of those solutions are.

In one sense the challenges are nothing new. Particle and space physicists have been doing team science for decades. But many of those enterprises grew up around major facilities or dedicated institutions. Their goals were clear at the outset, and structures, processes and cultures developed accordingly. Furthermore, the disciplines involved - physics, astronomy, engineering and computation - are far from alien to one another.

Now fundamental biology and biomedical research are more and more facing similar challenges. But the teams are emerging within frameworks established for a more traditional ethos of investigatorand hypothesis-driven research. What is more, progress requires that biologists, chemists, physicists and engineers mingle and even merge their disciplinary cultures and languages - sometimes an extremely tall order.

Whether in genomics, chemical biology, nanotechnology or imaging for cancer research, the case studies at the NIH meeting time and again highlighted the need for good communication across the collaborations - weekly video meetings over the web seemed a minimal requirement for success. And personality is everything. "Pick people you can rely on," said one leader. "You cannot regulate for personality," said another, "but you can foster generosity of spirit."

Predictably, many of the recommendations were aimed at the NIH. And so they should be: the agency has identified team science as a key element of its 'road map', whereas, as the meeting frequently highlighted, its rules either obstruct team science or do too little to facilitate it. The meeting's conclusions (see www.becon.nih.gov/ symposium 2003.htm) now need to be followed through with the full support of those at the top in the NIH.

\section{Essentials}

Some golden rules emerged for team leaders. First, do not underestimate the demands of running the team. Even the simplest collaboration might require two principal investigators, two department chairs and two deans to reach agreements. Several relatively new major interdisciplinary centres highlight the importance of $\mathrm{PhD}$ administrators - scientists or ex-scientists who are responsible for ensuring that recruitment, grant applications and similar key tasks proceed in good order, while actively contributing to the development of the scientific programme. The lack of an adequate career track for such individuals and project leaders more generally was highlighted.

Another golden rule is to establish certain key principles at the outset of a collaboration. Take technology transfer. Not only should differences in disciplinary, institutional and even national practices be addressed in negotiations at the outset, but university technologytransfer departments should be closely involved from the start and treated as part of the team, in order to minimize delays and obstacles further down the road. Bear in mind, said one participant, that technology-transfer revenues amount to only a few per cent of most institutions' income, and that there are very simple model agreements that can be applied in most cases, minimizing the need to reinvent wheels.

Another key aspect to be negotiated at the outset of a collaboration is the inescapable need for principles concerning team publications. Many collaborations progress splendidly, only to come to blows when it becomes necessary to list authors on a publication. The meeting highlighted how useless is the list of authors as a measure of their contributions, and urged journals to allow authors to publish lists of their respective contributions to a paper. Nature and its associated journals already do this, but will take further steps to encourage it, for example by including fields for such information in the electronic submission template. Indeed, many at the meeting urged that we and other journals should make such information compulsory. Only journal editors at the meeting expressed reservations on this. Readers are invited to send in their views.

\section{Due recognition}

But the big theme that emerged time and again related to the inability, because of the way science is currently done, to give credit and recognition to scientists who are part of a team. For example, too often, teams aim for a few high-profile publications that cover a lot of ground in a highly condensed manner. These miss opportunities not only to spell out interesting technical developments achieved along the way, but also to ensure that the people who delivered such innovations get any external recognition for them. Team leaders need to pay more attention to fostering additional publications in specialized journals, not as 'salami slices', but as appropriately focused accounts of genuinely innovative developments in techniques.

But the biggest challenge highlighted in the discussions is a scandal too often dismissed as insoluble: the way in which universities' appointments, promotion and tenure committees work or, more to the point, fail to work. The list of problems is a long one. Too often their considerations are overly systematized, so that consideration of candidates is inappropriately swayed by quantitative measures such as the impact factors of journals in which the candidate has published, the quantity of publications and whether or not the candidate was a first author. Too often the committees involve, say, history professors when deciding on the merits of a scientist whose multidisciplinary involvement makes him or her a challenge for even scientists to assess. Such a system is too clumsy to do justice to what should be crucial decisions for universities: the appointment of the people who will be their lifeblood. Yet, at the meeting at least, any idea that such committees could change their practices was dismissed out of court.

Given this and other difficulties in achieving due recognition, it is hard to see why anyone would want to become a team scientist. But as the meeting showed, funders, journals, universities and teams themselves can and should all take concrete steps to enhance their flexibility and to make the doing of team science as attractive to young researchers as the scientific challenges that necessitate it. 